\title{
Persistent measles virus infection of the intestine: confirmation by immunogold electron microscopy
}

\author{
J Lewin, A P Dhillon, R Sim, G Mazure, R E Pounder, A J Wakefield
}

\begin{abstract}
This study sought to investigate persistent measles virus infection of the intestine: a novel protocol for immunogold electron microscopy was developed using a polyclonal anti-measles nucleoprotein antibody on reprocessed, formalin fixed paraffin wax embedded tissue sections. Antibody binding was detected using both immunoperoxidase and light microscopy on tissue sections, and $10 \mathrm{~nm}$ gold conjugated secondary antibody and electron microscopy on ultrathin sections. The techniques were validated using both measles infected vero cells and human tissues with established measles infection: these included brain affected by subacute sclerosing panencephalitis and acute measles appendicitis. The technique was applied subsequently to six untreated cases of granulomatous Crohn's disease, and two cases of ileocaecal tuberculosis, a granulomatous control. Mumps primary antibody - applied to both mumps infected vero cells, and measles infected vero cells and tissues studied by immunoperoxidase, and measles antibody on mumps infected cells studied by immunoperoxidase and immunogold were used as specificity controls: the primary antibodies identified their respective target antigen and there was no antibody cross reactivity. Measles virus nucleocapsids labelled with gold conjugated antibody in both infected cells and tissues, including foci of granulomatous inflammation in five of six cases of Crohn's disease: in the fifth case, the granuloma could not be identified in ultrathin section. In one of the tuberculosis cases, a low level of signal was noted while the second case was negative. Labelling adopted a characteristic pattern in all infected tissues, strengthening the specificity of these findings. This study provides the first direct confirmation of persistent measles virus infection of the intestine.
\end{abstract}

(Gut 1995; 36: 564-569)

Keywords: measles virus, intestine, immunogold electron microscopy.

Work by our group ${ }^{1}$ and others ${ }^{2}$ has provided preliminary evidence of persistent measles virus infection in the gastrointestinal tract: data, derived from a combination of ultrastructural analysis, in situ hybridisation, and immunohistochemistry, suggest that persistent infection is a feature, particularly of Crohn's disease, and that it can be identified within foci of granulomatous and lymphocytic inflammation, phenotypic characteristics of this condition. Recent epidemiological studies from Sweden ${ }^{3}$ linked early measles virus infection to the development of Crohn's disease in later life, a scenario that would be analagous to subacute sclerosing panencephalitis. ${ }^{4}$

Persistent paramyxovirus infection has been implicated in a number of diseases of unknown aetiology, sometimes based upon ultrastructural findings. ${ }^{5}$ High resolution images of paramyxovirus like particles should be interpreted with extreme caution because the nucleocapsids that are seen characteristically, in for example subacute sclerosing panencephalitis, may be confused with a number of non-viral structures. ${ }^{56}$

This study sought to confirm the viral origin of the nucleocapsid like particles that we have seen in Crohn's disease, by colocalising these particles with gold labelled antibody specific for measles virus nucleocapsid protein. Despite an intensive ultrastructural survey we have been unable to identify similar particles in either normal intestine, or intestine affected by ulcerative colitis. Therefore, this study sought to confirm measles virus persistence in gut affected by Crohn's disease. It was not designed to investigate an aetiological role for the agent in this condition.

\section{Methods}

\section{Cell culture}

Vero (green monkey kidney) cells were cultured in $75 \mathrm{~mm}$ flasks using standard techniques. Confluent monolayers were infected with either Edmonston strain measles virus at a titre of $10^{7}$ plaque forming units per $\mathrm{ml}$, or mumps virus (Taylor strain: PHLS, Colindale, UK) at a $\mathrm{TCID}_{50}$ (Tissue Culture Infectious Dose) of $10^{4}$; uninfected cells were used as a negative control. When $>80 \%$ of cells showed cytopathic effect, monolayers were fixed using $1 \%$ glutaraldehyde $/ 1 \%$ paraformaldehyde in phosphate buffered saline for 30 minutes. The cells were scraped from the flask using a platinum wire loop and the suspended cells were pelleted in Eppendorf tubes using a Beckman microfuge (Palo Alto, CA, USA). Cell pellets were washed after fixation with phosphate buffered saline and dehydrated using dimethylformamide, 50,70 , and $90 \% \times 2$ changes each of 10-20 minutes duration depending on the size of the block. The blocks were then infiltrated with a 50:50 


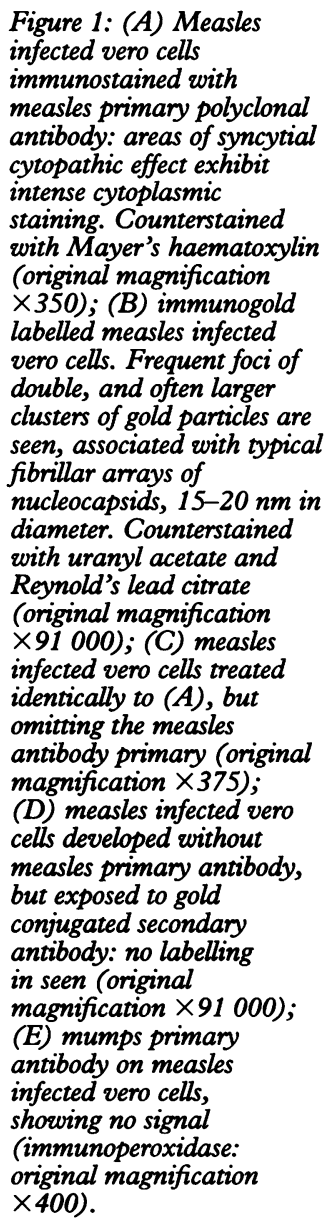

Figure 1: (A) Measles infected vero cells immunostained with measles primary polyclonal antibody: areas of syncytial cytopathic effect exhibit intense cytoplasmic staining. Counterstained with Mayer's haematoxylin (original magnification $\times 350$ ); (B) immunogold labelled measles infected vero cells. Frequent foci of double, and often larger clusters of gold particles are seen, associated with typical fibrillar arrays of

nucleocapsids, 15-20 $\mathrm{nm}$ in diameter. Counterstained with uranyl acetate and Reynold's lead citrate (original magnification $\times 91000)$; (C) measles infected vero cells treated identically to $(A)$, but omitting the measles antibody primary (original magnification $\times 375$ ); (D) measles infected vero cells developed without measles primary antibody, but exposed to gold conjugated secondary antibody: no labelling in seen (original magnification $\times 91000$ ); (E) mumps primary antibody on measles infected vero cells, showing no signal (immunoperoxidase: original magnification $\times 400)$.
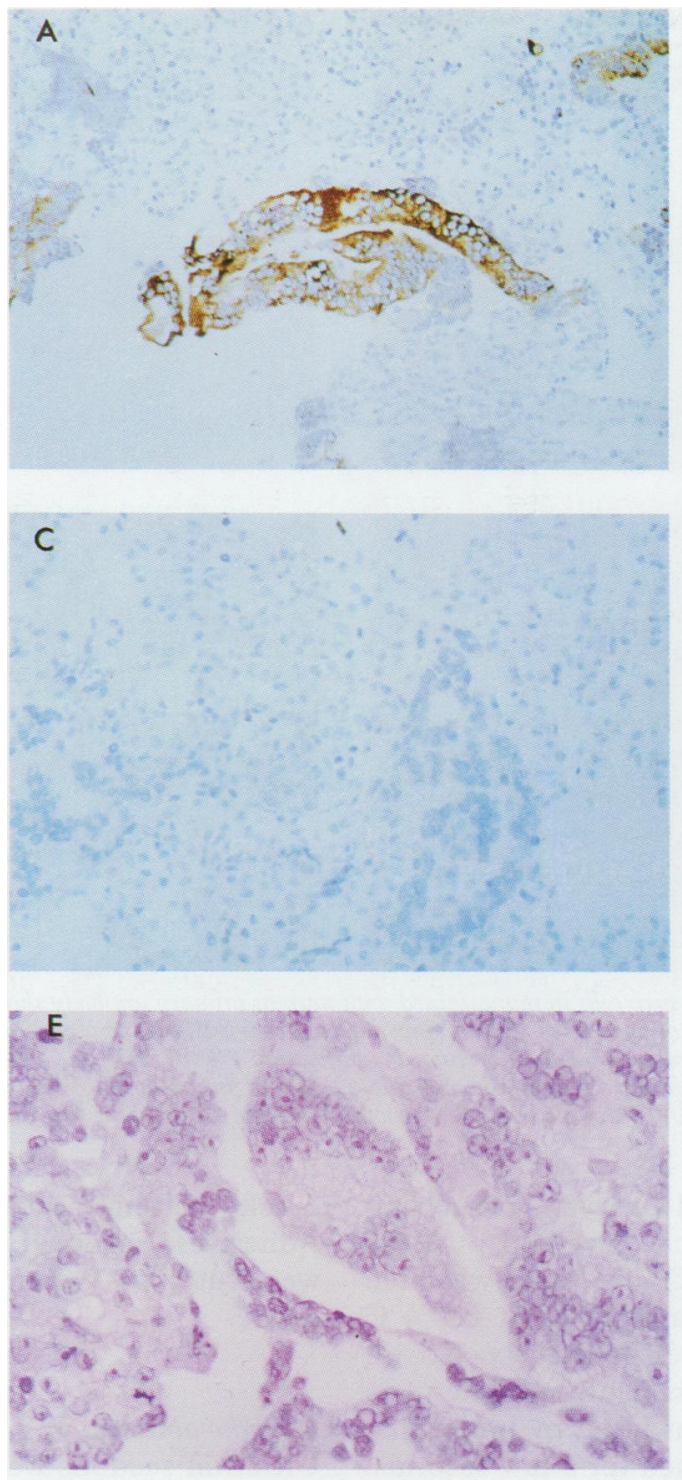

mixture of dimethylformamide and LR White resin with $0.5 \%$ benzoin photoinitiator (TAAB Laboratories Equipment, Reading, UK) added, for $30-60$ minutes, followed by $100 \%$ resin for one to two hours. The blocks were embedded in closable embedding capsules using fresh LR White with photoinitiator. Polymerisation was carried out at $4^{\circ} \mathrm{C}$ using an ultraviolet light source at $10 \mathrm{~cm}$ distance. The resin polymerised in one to two hours and the small amount of resin remaining unpolymerised under the closure was removed using a cotton swab.

Tissue reprocessed from paraffin blocks Initially, archival formalin fixed paraffin wax processed tissues with established measles infection were selected: these included cerebral tissue from a case of subacute sclerosing panencephalitis, and appendix from a case of acute measles virus appendicitis (gift of Dr H Reid, Chase Farm Hospital, Enfield). Six cases of granulomatous Crohn's disease were selected that included four rectal biopsy specimens and two appendixes taken at the time of initial presentation (that is, before specific or immunosuppressive therapy was instituted). In both the positive controls and Crohn's disease
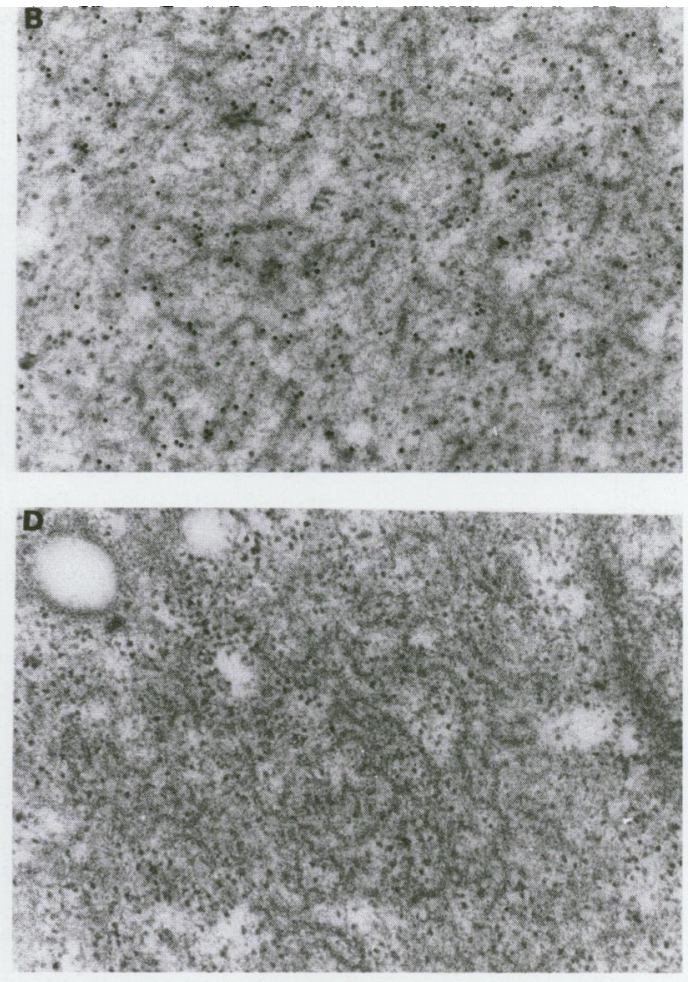

tissues, the diagnoses were established by standard clinical and histopathological criteria. Two cases of ileocaecal tuberculosis were studied as granulomatous controls. Sections were cut from tissue blocks and immunostained with a polyclonal antibody specific for the measles virus nucleocapsid protein (gift of J Stephenson and T Fookes, CAMR, Porton Down, UK) using an immunoperoxidase technique, as described previously. ${ }^{1}$ Serial tissue sections were processed with either omission of the primary antibody or exposure to mumps (a related paramyxovirus) specific monoclonal antibody (Seralab, Crawley, Sussex, UK) as negative controls. Cross reactivity of the measles and mumps specific antibodies was examined in vero cells infected with these viruses, before the immunogold studies.

\section{Processing for electron microscopy}

Suitable areas of tissue were selected for immunogold analysis: these included areas that gave positive measles staining by immunoperoxidase, and foci of granulomatous inflammation in the cases of Crohn's disease and ileocaecal tuberculosis. Wedges of tissue were removed from the paraffin wax block using a single edged razor blade. The pieces were dewaxed with chloroform followed by rinsing in absolute alcohol. The tissue was then infiltrated with resin and embedded as described above.

Paraffin wax sections only were available on the acute measles appendicitis. A novel method was used to lift the tissue from the slide: the section was dewaxed and taken to absolute alcohol, it was then flooded with 50:50 mixture of alcohol, and LR White resin with photoinitiator added for a further 15 minutes; it was then drained and the slide blotted to remove surplus resin. Drops of 

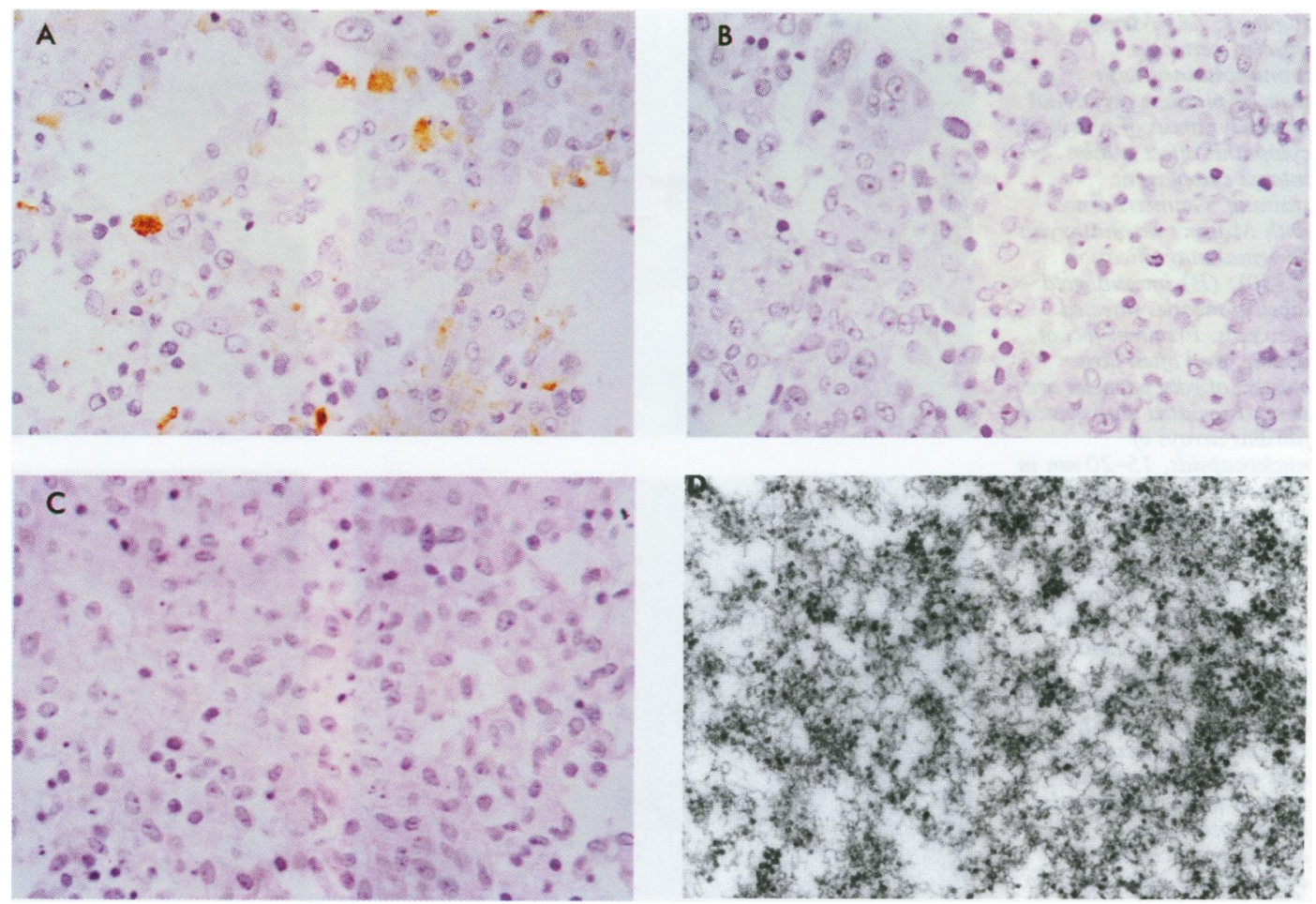

Figure 2: (A) Mumps infected vero cells immunostained with mumps primary antibody showing cytoplasmic staining in infected cells (original magnification $\times 400$ ); (B) mumps infected vero cells treated identically to $(A)$, but omitting the mumps primary antibody (immunoperoxidase: original magnification $\times 400) ;(C)$ and (D) measles primary antibody on mumps infected vero cells examined by immunoperoxidase $(C)$ (original magnification $\times 400$ ) and immunogold (D) (original magnification $\times 82400$ ). No antibody cross reactivity is seen. In (D), the fine fibrillary arrays of mumps nucleocapsids are seen clearly.

fresh resin were placed over the tissue and covered with plastic coverslips (Agar Scientific, Stansted, UK). The resin was then polymerised with ultraviolet light at $4^{\circ} \mathrm{C}$ for one hour. The coverslip was peeled off and unpolymerised resin removed. The resin containing the tissue was lifted by immersing the slide in liquid nitrogen for a few seconds. The pieces were then trimmed and mounted onto blank resin blocks using Permabond

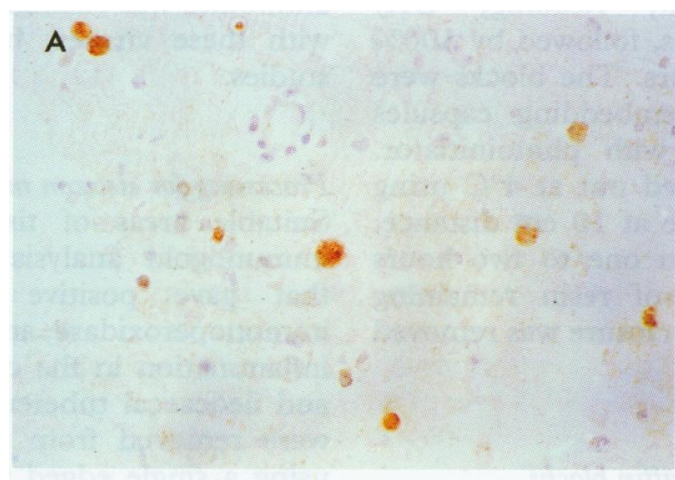

D quick setting epoxy resin (Permabond Adhesives, Eastleigh, UK). Ultrathin sections were cut at $50-80 \mathrm{~nm}$ and immunostained as above.

\section{Immunolabelling}

Ultrathin sections were cut at 70-80 $\mathrm{nm}$ and picked up onto Pioloform (Agar Scientific, Stansted, UK) coated 300 mesh nickel grids

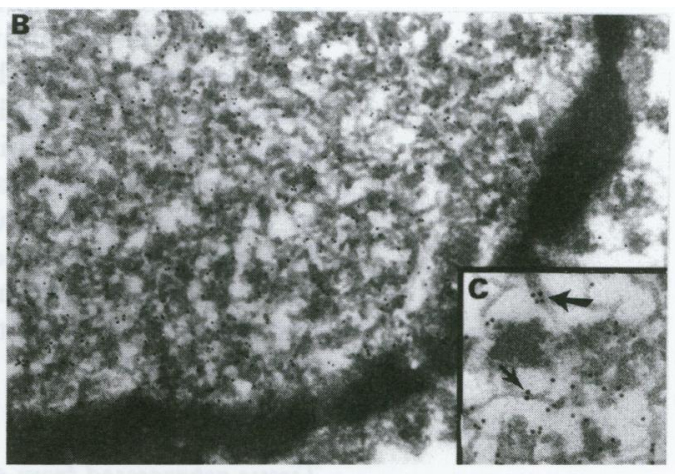

Figure 3: Subacute sclerosing panencephalitis:

immunostaining for measles virus nucleocapsid protein in subacute sclerosing panencephalitis brain examined by light microscopy $(A)$ (original magnification $\times 400$ ) and electron microscopy (B) and (C). Intranuclear nucleocapsids are seen in (B), frequently showing paired gold particles spanning individual nucleocapsids, shown clearly in (C), showing cytoplasmic signal (original magnifications; (B) $\times 62500$, (C) $\times 95000)$. No signal is seen in identically processed tissue sections, in which the primary measles antibody was omitted (D); immunoperoxidase original magnification $\times 400$ ). 

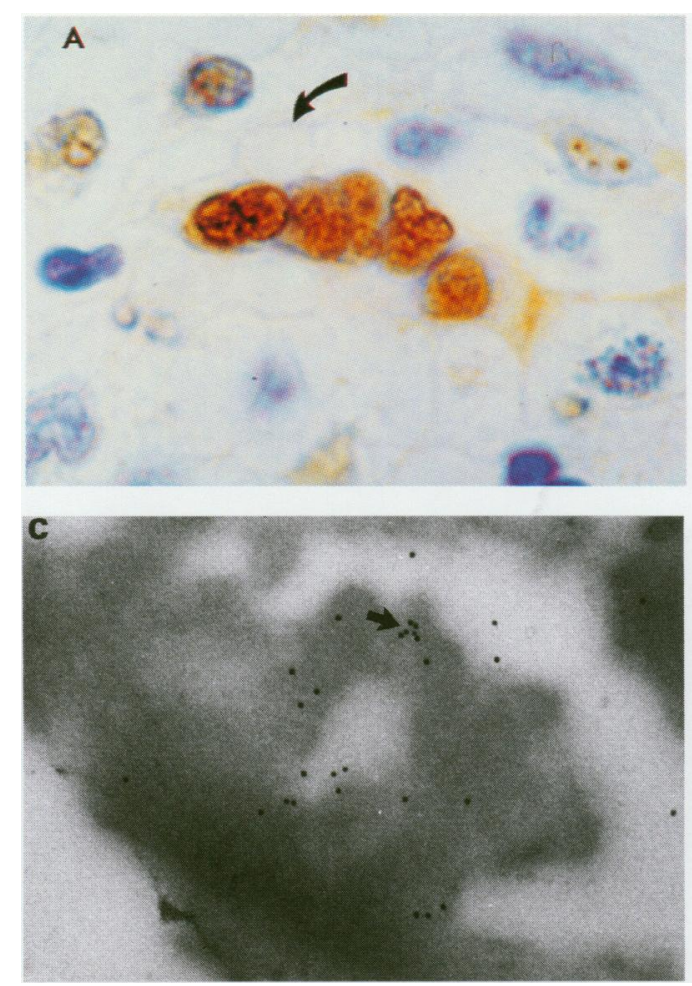

(Gilder, Grantham, UK). The grids were incubated on drops of $5 \%$ normal goat serum in $0.1 \%$ bovine serum albumin/phosphate buffered saline buffer for 30 minutes, they were then transferred to drops of primary measles polyclonal antibody $1 / 100$ in $0.1 \%$ bovine serum albumin/phosphate buffered saline for one hour. Primary antibody was removed by washing on drops of phosphate buffered saline $5 \times 5$ minutes, the first transfer being made without removing any surplus liquid from the grid. Surplus buffer was removed on the remaining four changes by touching the edge on a piece of blotting paper, taking care not to allow the grid to dry out. The grids were then transferred to drops of gold conjugate (Biocell, Cardiff, UK) diluted 1/100 with phosphate buffered saline for one hour followed by washing with drops of phosphate buffered saline $2 \times 5$ minutes to remove unbound gold conjugate and a final rinse in distilled water. Sections were stained lightly with uranyl acetate and Reynold's lead citrate and viewed using a Philips 201 transmission electron microscope. Sections processed omitting the primary antibody, were included with all tissues as negative controls.

\section{Results}

Measles infected vero cells

Vero cells that had been experimentally infected with measles virus exhibited positive immunoperoxidase and immunogold labelling (Fig 1A and B), which was not seen either in identically processed measles infected cells not exposed to the primary measles antibody (Fig 1C and D), or in measles infected cells exposed to the mumps primary antibody (Fig 1E). Ultrastructurally, viral nucleocapsids

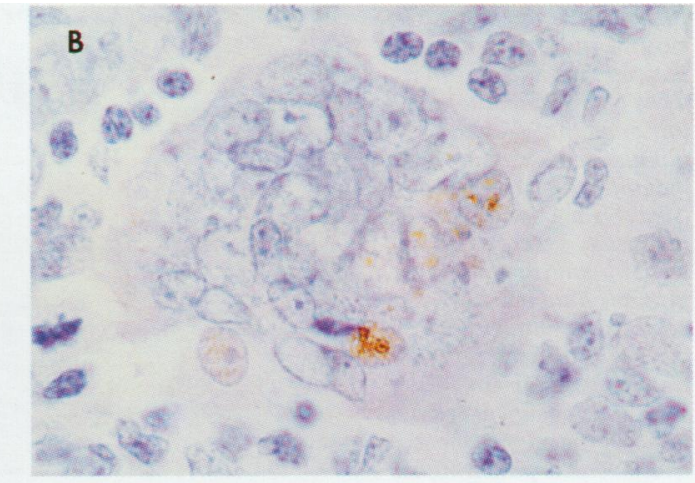

Figure 4: Measles appendicitis: immunoperoxidase treated sections stained for measles virus $N$ protein. Positive signal is seen in an endothelial cell syncytium (A) (erythrocytes arrowed) (original magnification $\times 1000$ ) and $a$ Warthin Finkeldey giant cell $(B)$ (original magnification $\times 800$ ). Immunogold labelling was seen in an identical cellular location despite suboptimal preservation $(C)$. The section was not counterstained because the dark contrast obscured the gold particles on other sections of the same case. A cluster of apparently 'paired' gold particles is arrowed - a similar image is seen later in Figs $5(E)$ and $(F)$.

(Original magnification $\times 111375$.)

consisted of characteristic parallel fibrillar structures of between 15 and $20 \mathrm{~nm}$ diameter.

A characteristic pattern of labelling was seen in both nucleus and cytoplasm of infected cells that consisted of gold particles - grouped, often in pairs, and bound at discrete points along the viral nucleocapsids (Fig 1B): this pattern was seen consistently in both infected cells and infected tissues, and was not seen in the very occasional background signal seen in control sections. Mumps infected vero cells stained strongly by immunoperoxidase using the primary mumps antibody (Fig 2A), but did not stain when the mumps primary antibody was omitted in otherwise identically processed cells (Fig 2B). No signal was seen when the measles antibody was put onto mumps infected cells at either the light microscopic or ultrastructural levels (Fig 2C and D).

\section{Subacute sclerosing panencephalitis}

Strong signal for measles virus was seen predominantly in the nuclei of infected cells, using both the immunoperoxidase technique on histological sections (Fig 3A), and immunogold labelling of ultrathin sections of infected brain (Fig 3B and C). Ultrastructurally, viral nucleocapsids were well preserved despite suboptimal fixation and paraffin processing. Again, the double immunogold signal on nucleocapsids was a consistent feature (Fig 3B and C). No signal was seen on sections in which the primary measles antibody had been omitted, either at the light microscopic (Fig 3D), or ultrastructural levels (data not shown).

\section{Intestinal tissues}

The techniques were applied subsequently to measles infected intestinal tissue - acute infected measles appendicitis - with similar 
Figure 5: Crohn's disease: positive nuclear staining in macrophage like cells in a focus of granulomatous inflammation $((A)$; immunoperoxidase: original magnification $\times 600$ ). Signal is not seen in an identically processed serial section, omitting the primary antibody ((B), original magnification $\times 600$ ). Positive immunogold labelling is seen in an identical cellular location and shows the characteristic paired signal (arrowed) ((C), original magnification $\times 71000)$. ((D) (inset) shows a high power view of gold label distributed along the length of what appears to be a single nucleocapsid: original magnification $\times 139$ 000); ((E) nuclear staining in a macrophage in a focus of granulomatous inflammation from another case of Crohn's disease. The paired signal is seen, particularly in the central cluster (ringed), shown magnified in $(F)$, this shows clearly, the parallel arrays (arrowed) of a

$17 \mathrm{~nm}$ diameter nucleocapsid spanned by the gold label, original magnifications:

(E) $\times 91000,(F) \times$ 95 000). Staining was not seen in otherwise identically processed sections of

Crohn's disease when the measles primary antibody was omitted ((G) original magnification $\times 54$ 000).
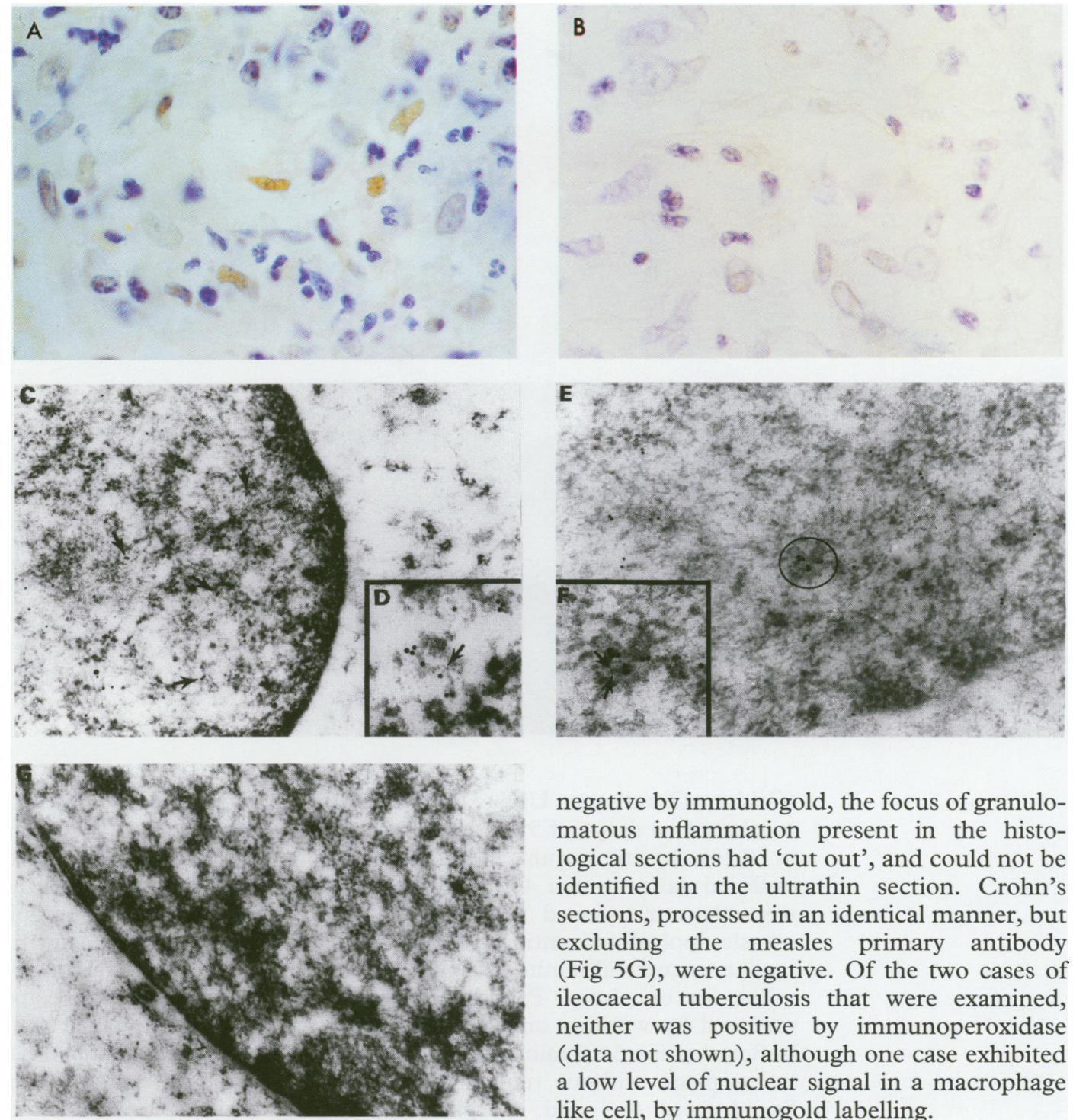

negative by immunogold, the focus of granulomatous inflammation present in the histological sections had 'cut out', and could not be identified in the ultrathin section. Crohn's sections, processed in an identical manner, but excluding the measles primary antibody (Fig 5G), were negative. Of the two cases of ileocaecal tuberculosis that were examined, neither was positive by immunoperoxidase (data not shown), although one case exhibited a low level of nuclear signal in a macrophage like cell, by immunogold labelling.

results. Measles virus antigen was identified by the immunoperoxidase technique in endothelial cells (Fig 4A), and macrophage like cells including Warthin-Finkeldy giant cells (Fig 4B), a classic feature of acute measles virus infection, and occasional lymphocytes within lymphoid follicles. Staining was not seen in negative control sections. Immunogold labelling confirmed the presence of the virus in the same cellular foci despite suboptimal preservation (Fig 4C). No signal was seen in the absence of the primary antibody (data not shown).

Of the six cases of Crohn's disease examined, all were positive for measles by immunoperoxidase within cells consistent in morphology and location with histiocytic macrophages (Fig 5A), endothelial cells, and occasional lymphocytes: signal was not seen in sections that were either not exposed to the measles primary antibody (Fig 5B), or exposed to mumps primary antibody (data not shown). Five cases were positive by immunogold in the same cellular location, and viral particles exhibited the characteristic features of size, shape, and immunogold labelling described above (Fig 5C-F): in one case, signal was detected, additionally, in a single crypt epithelial cell. In the fifth case, which was

\section{Discussion}

This paper presents the first direct evidence of measles virus persistence in the intestine; that is, the colocalisation of a specific antibody with viral nucleocapsids at the ultrastructural level. The cellular localisation of measles virus in Crohn's tissues is consistent with that seen previously using different techniques, ${ }^{12}$ and in different laboratories. ${ }^{2}$ The colocalisation of a specific antibody with particles that are, in terms of both size and morphology, identical to the target virus, is strong evidence for the presence of that virus: the characteristic pattern of labelling of nucleocapsids with gold particles provided a further, if unexpected, degree of specificity. The detection of measles in one of two cases of ileocaecal tuberculosis raises the possibility that persistently infected immune cells aggregate in foci of inflammaof the granuloma. This notwithstanding, in Crohn's disease tissues, the detection of measles virus within resident intestinal cells, including endothelium and epithelium, does suggest persistent intestinal infection. It is possible, however, that circulating immune cells within the same host may also be persistently infected. tion, and are unrelated to the primary cause 
Cortocosteroids, a mainstay in the treatment of patients with Crohn's disease, may predispose to permissive viral replication, and confound the question of persistence within the inflamed intestine: none of the patients whose tissues were examined in this study had received either corticosteroids or other immunosuppressive treatment.

Previous work by our group provided evidence for occasional persistent measles virus infection both in non-inflamed intestine, and in patients with conditions other than Crohn's disease. ${ }^{1}$ It has been proposed that persistent infection is established frequently during acute measles virus infection, and that ensuing lifelong immunity is maintained by constant antigenic stimulation. ${ }^{7}$ Frequent environmental re-exposure, an alternative explanation, is not a prerequisite for continued immunity. ${ }^{78}$ If, as is suggested by our studies, the intestine is a comparatively common site of virus persistence, then constant antigenic stimulation must be balanced with tolerance in most subjects. Future research will seek to discover if the Crohn's granuloma is a focus wherein such immunological tolerance has been breached.

In summary, we have validated a novel protocol for using immunogold electron microscopy for the detection of both acute and persistent measles virus infection in formalin fixed paraffin wax processed tissues. We have used this technique to confirm persistent measles virus infection of intestinal tissues affected by Crohn's disease. A large comparative study of intestinal tissues is now underway to determine the relative specificity of these findings for Crohn's disease. The applicability of this technique to routinely processed specimens raises exciting possibilities for the investigation of diseases in which a role for persistent measles virus infection has been implicated.

We are grateful to the following for their valuable assistance: Drs J Stephenson and T Fookes, CAMR, Porton Down, UK for the provision of measles polyclonal antibody, Dr $\mathrm{H}$ Reid, Chase Farm Hospital, Enfield, for the measles appendicitis tissues, Francis Moll for the photography, and Nilanthe Kelsey for preparation of the manuscript. This work was supported by the National Association for Colitis and Crohn's disease.

1 Wakefield AJ, Pittilo RM, Sim R, Cosby L, Stephenson JR, Dhillon AP, et al. Evidence of persistent measles virus infection in Crohn's disease. $f$ Med Virol 1993; 39: 345-53.

2 Knibbs DR, van Kruiningen, Colombel JF, Cortot A. Ultrastructural evidence of paramyxovirus in two french families with Crohn's disease. Gastroenterology 1993; 104: A726.

3 Ekbom A, Wakefield AJ, Zack M, Adami HO. Perinatal measles infection and subsequent Crohn's disease. Lance 1994; 344: 508-10

4 Miller C, Farringdon CP, Harbert K. The epidemiology of subacute sclerosing panencephalitis in England and Wales 1970-1989. Int f Epidemiol 1992; 21: 998-1006.

5 Jenyung L, Bockus D, Remington F. Tuboreticular structures and cylindrical confronting cysternae. Hum Pathol 1989; 20: 617-27.

6 Grimley PM, Schaff F. Significance of tuboreticular inclusions in the pathobiology of human diseases. Pathobiol Annu 1976; 6: 221-57.

7 ter Meulen V, Carter M. Measles virus persistency and disease. Prog Med Virol 1984; 30: 44-61.

8 Panum PL. lagttageiser anstillede under maeslingeapidemien paa faerorae i aaret 1846 . Bibltk Laeger $1847 ; 1$ 270-344. 